Revue d'histoire de l'Amérique française

ZAS REVUE D.HISTOIRE DE L'AMÉRIQUE FRANÇAISE

\title{
L'organisation militaire sous le régime britannique et le rôle assigné à la gentilhommerie canadienne (1760-1815)
}

\section{Roch Legault}

Volume 45, numéro 2, automne 1991

URI : https://id.erudit.org/iderudit/304967ar

DOI : https://doi.org/10.7202/304967ar

Aller au sommaire du numéro

Éditeur(s)

Institut d'histoire de l'Amérique française

ISSN

0035-2357 (imprimé)

1492-1383 (numérique)

Découvrir la revue

Citer cet article

Legault, R. (1991). L’organisation militaire sous le régime britannique et le rôle assigné à la gentilhommerie canadienne (1760-1815). Revue d'histoire de l'Amérique française, 45(2), 229-249. https://doi.org/10.7202/304967ar
Résumé de l'article

L'étude du contexte dans lequel l'élite militaire du régime français allait être plongée après 1760 permet d'observer les difficultés que posent l'amorce ou la poursuite d'une carrière militaire pendant le régime britannique pour la gentilhommerie canadienne. Prenant en considération les avantages sociaux que pouvaient retirer les officiers et leurs familles des différentes organisations militaires, tant sous le régime français que sous le régime anglais, notre approche débouche finalement sur des interrogations propres à l'histoire sociale. 


\title{
L'ORGANISATION MILITAIRE SOUS LE RÉGIME BRITANNIQUE ET LE RÔLE ASSIGNÉ À LA GENTILHOMMERIE CANADIENNE (1760-1815)
}

\author{
ROCH LEGAULT ${ }^{1}$ \\ Département d' histoire \\ Université de Montréal
}

\section{RÉSUMÉ}

L'étude du contexte dans lequel l'élite militaire du régime français allait être plongée après 1760 permet d'observer les difficultés que posent l'amorce ou la poursuite d'une carrière militaire pendant le régime britannique pour la gentilhommerie canadienne. Prenant en considération les avantages sociaux que pouvaient retirer les officiers et leurs familles des différentes organisations militaires, tant sous le régime français que sous le régime anglais, notre approche débouche finalement sur des interrogations propres à l'histoire sociale.

\begin{abstract}
By studying the circumstances of the military elite of the French regime after the Conquest, it is possible to understand the difficulties the Canadian gentry faced starting or pursuing a military career under British rule. Taking into account the social benefits that accrued to the officers and their families, whether under the French or British regime, from their participation to the various military organizations, this inquiry broadens the traditional field of military history by emphasizing the social context of military experience.
\end{abstract}

Dans cet article, nous présentons l'organisation de défense du Canada mise en place par les autorités britanniques après 1760, sans prétendre bien sûr épuiser le sujet. Nous tentons ensuite de montrer comment l'ancienne élite militaire du régime français vit un bouleversement institutionnel, c'est-à-dire un passage d'une structure de défense où on lui ménage une place importante à une autre où elle ne joue plus qu'un rôle de second ordre. Après un survol de l'organisation militaire de la Nouvelle-France, nous abordons l'évolution du

1 Nous remercions les professeurs Louise Dechêne et Pierre Tousignant ainsi que Lorraine Gadoury et Daniel Massicotte pour leurs précieux commentaires et suggestions. 
système de défense pendant le régime britannique, qui repose principalement sur l'armée régulière et, secondairement, sur les forces proprement nord-américaines: corps de volontaires, milices incorporées et territoriaux. Enfin, nous examinerons le nouveau rôle assigné aux anciennes familles militaires du régime français dans cette structure.

Sans entrer dans les débats à propos de la définition d'une élite, d'une gentilhommerie ou d'une noblesse canadienne, nous avons retenu les noms qui figurent sur la liste des nobles que le gouverneur Guy Carleton a fait dresser en $1767^{2}$. Ces individus forment le noyau central de l'élite militaire de la colonie selon leurs contemporains.

Jusqu'ici l'historiographie de la période pré-industrielle, sans négliger le rapport étroit qu'entretient la gentilhommerie canadienne avec les institutions militaires, n'a pas poursuivi la recherche et la réflexion sur ce terrain. C'est ainsi que Fernand Ouellet, qui plus que tout autre historien s'est intéressé au sort de la noblesse après la Conquête, avance que la faiblesse de ce groupe social est d'abord due à son incapacité à s'adapter au nouveau contexte économique ${ }^{3}$ et notamment à faire face aux assauts de la bourgeoisie dans le domaine de la propriété seigneuriale. Même s'il écrit que la noblesse canadienne possède d'abord une vocation militaire sous le régime français $^{4}$, il s'intéresse peu aux différences des institutions militaires ${ }^{5}$. C'est précisément ce facteur, qui n'entre pas dans l'interprétation de Fernand Ouellet, soit l'effet de la déstructuration de l'univers institutionnel militaire de la Nouvelle-France sur la gentilhommerie canadienne, qui fait l'objet de notre enquête. Celle-ci diffère donc des perspectives généralement adoptées par les historiens québécois depuis les années 1960 parce qu'elle privilégie l'étude de la gentilhommerie en relation avec les institutions, le politique et le militaire. Elle s'éloigne des considérations économiques qui retiendraient surtout le lien qu'entretient avec la terre le groupe à l'étude.

2 "A General State of the Canadian Noblesse, Actually in the Province of Quebec», Report of the Canadian Archives, 1888 (Ottawa, 1889), 44-48. Ce document a été présenté par Fernand Ouellet dans une note de recherche intitulée "La noblesse canadienne" en 1767: un inventaire», Histoire sociale/Social History, 1,1 (1968): 129-137.

3 Les travaux de Lorraine Gadoury sur la noblesse n'abordent pas comme tel l'aspect militaire. Voir son mémoire de maîtrise, Une famille noble en Nouvelle-France: les D'Ailleboust, Université de Montréal, 1982; et sa thèse de doctorat, Comportements démographiques et alliances de la noblesse en Nouvelle-France, Université de Montréal, 1988.

4 Fernand Ouellet, «Propriété seigneuriale et groupes sociaux dans la vallée du SaintLaurent (1663-1840)», La revue de l'Université d'Ottawa, 47,1-2 (1977): 186.

5 Préoccupé par la volonté de masquer les ruptures engendrées par le changement de régime, Fernand Ouellet ignore, même dans son enquête sur la milice, cet effet déstructurant sur la gentilhommerie. «Officiers de milice et structure sociale au Québec (1660-1815)», Histoire sociale/Social History, 12,23 (1977): 36-65. 


\section{L'ORGANISATION MILITAIRE DE LA NOUVELLE-FRANCE}

L'armée de terre française ne fait pas véritablement partie de l'organisation militaire permanente de la Nouvelle-France. Elle n'intervient qu'en 1665 pour donner une correction aux Iroquois (le régiment de Carignan-Salières) et dans la guerre de Sept Ans pour contrer l'armée régulière britannique, envoyée en force en Amérique du Nord. Quelques Canadiens bénéficient de commissions d'officiers à cette dernière occasion.

C'est le ministère de la Marine, responsable des colonies, qui a charge de la protection du Canada. Il achève de mettre au point l'organisation militaire de la Nouvelle-France vers les années 1680-1690. En effet, en 1683, des troupes réglées de la Marine prennent place aux côtés de la milice canadienne pour constituer les forces de défense permanentes de la colonie. Elles deviendront les compagnies franches de la Marine. Il importe ici de souligner la différence entre ces deux institutions, la milice et les compagnies franches, quant au statut et aux fonctions de leurs officiers.

À la fin du régime français, les officiers de milice «sont davantage des agents de l'administration que des commandants militaires ${ }^{6}$. Ils secondent l'intendant et le grand voyer, agissent à l'occasion comme syndic et arbitrent même des litiges entre habitants et communautés rurales. En somme, l'officier de milice, doit être considéré avant tout comme «un agent de l'État» et non comme un militaire ${ }^{7}$. En cas de guerre, le milicien et ses supérieurs se transforment en soldats, certes, mais les conditions de participation au combat diffèrent de celles des troupes de la colonie (les compagnies franches de la Marine). Officiers et miliciens ne reçoivent aucune solde pour leurs services, ni même un uniforme complet. La commission d'officier de milice ne confère à son détenteur que le droit de commander uniquement des miliciens tandis que des sous-officiers et des cadets de l'armée de la Marine ${ }^{8}$ se retrouvent à la tête des milices menées au front. Enfin, fait significatif, très peu d'officiers de milice arborent la Croix de Saint-Louis ${ }^{9}$, la décoration du mérite militaire en Nouvelle-France.

6 Christopher J. Russ, Les troupes de la Marine, 1683-1713, mémoire de maîtrise (histoire), Université McGill, 1971, 55.

7 Fernand Ouellet, "Officiers de milice et structure sociale au Québec», 42-43.

8 Gustave Lanctôt, "Les troupes de la Nouvelle-France», The Canadian Historical Report (Ottawa, Department of Public Archives, 1926), 41. Voir aussi l'étude récente de Jay Cassel, Les troupes de la Marine, 1683-1760, Men and Materials, thèse de doctorat (histoire), Université de Toronto, 1987.

9 Un seul selon Aegidius Fauteux, Étienne Charest (Chevaliers de Saint-Louis en Canada (Montréal, Les Éditions des Dix, 1940), 204-205). Fernand Ouellet en mentionne un second, Dufy Desauniers, op. cit., 43. 
Nonobstant les privilèges et le statut social dont jouit l'officier de milice et sa présence dans les affaires civiles autant que militaires, celui qui désire entreprendre une véritable carrière des armes et profiter au maximum des largesses de l'État doit nécessairement opter pour les compagnies franches de la Marine.

En 1687, le gouverneur Denonville considère nécessaire pour le «service et l'intérêt public» d'obtenir les moyens de récompenser les officiers du Canada à son service de façon à se les attacher ${ }^{10}$. Les commissions dans les compagnies franches de la Marine vont être grandement convoitées par les familles coloniales et, dès 1702 , le contrôleur de la Marine Bacqueville de La Potherie suggère aux autorités métropolitaines de cesser l'envoi d'officiers français dans la colonie devant le mécontentement que cela suscite chez les Canadiens qui craignent pour leur avancement ${ }^{11}$. Bientôt, les premiers-nés des familles de l'élite sociale canadienne embrassent en grand nombre la carrière des armes ${ }^{12}$.

À la fin du régime français, l'effet conjugué de l'organisation des troupes du ministère de la Marine et des politiques de la métropole font en sorte de donner une place de choix dans les troupes de la Marine aux officiers canadiens ${ }^{13}$. À l'abri de la concurrence des officiers de la métropole dans les compagnies franches de la Marine, la gentilhommerie coloniale utilise cette fonction militaire pour prospérer et pour consolider sa position sociale.

\section{L'ORGANISATION MILITAIRE DU RÉGIME BRITANNIQUE}

L'organisation militaire du Canada se complique avec le nouveau régime. L'armée régulière britannique assume l'ensemble des tâches reliées à la défense de la colonie et au maintien de l'ordre public. Après quelques hésitations du gouvernement métropolitain, la milice est rétablie quoiqu'elle soit appelée à jouer un rôle très effacé. À cette milice et à l'armée régulière, s'ajoute une force d'appoint coloniale qui prend diverses formes entre 1775 et 1815 , et qui sert en quelque sorte de refuge à l'ancienne élite militaire de la Nouvelle-France.

10 George F. G. Stanley, Nos soldats, l' histoire militaire du Canada de 1604 a nos jours (Montréal, 1980), 57.

11 Ibid., 58.

12 Jay Cassel, The Troupes de la Marine in Canada, thèse de doctorat (histoire), Université de Toronto, 1988, 80-81.

13 Voir à ce sujet l'article de W. J. Eccles, «The Social, Economic and Political Significance of the Military Establishment in New France», The Canadian Historical Review, 52,1 (1971): 1-22. 
En effet, la milice ne peut pas servir de support économique à la noblesse désargentée et elle est incapable de répondre convenablement à ses aspirations sociales. Quant à l'armée régulière, elle lui est difficilement accessible, comme on peut le constater en observant la place occupée par la gentilhommerie canadienne dans les régiments britanniques de 1775 à 1815. Seules les familles D'Estimauville de Beaumouchel, Fleury Deschambault, d'Irumberry de Salaberry, Juchereau Duchesnay et Saint-Ours figurent dans la liste des officiers. Cette faible représentation nous invite à étudier d'abord la nature de l'armée et les politiques de ses dirigeants. Nous étudierons ensuite les solutions de rechange offertes aux gentilshommes canadiens: les milices et les corps provinciaux.

\section{A - L'armée britannique}

\section{1 - Caractéristiques générales}

Les historiens qui se sont penchés sur l'organisation de l'armée britannique sont unanimes à reconnaître la complexité de leur sujet d'étude. Les responsabilités importantes sont divisées et trop souvent, des organismes ou des individus se disputent au sujet du partage des tâches. Le "Commander in Chief», le «Master General of the Ordnance», le «Secretary at War», le «Secretary of State for War and Colonies», le «First Lord of Treasury» et quelquefois même le premier ministre s'occupent de l'administration de l'armée et du déroulement des opérations. L'historien britannique John Sweetman explique ainsi ce chaos apparent: le système de contrepoids caractéristique de l'appareil administratif britannique s'applique également à l'armée ${ }^{14}$. Il faut aussi y voir l'expression des luttes sociales et politiques. Depuis Jacques II et Cromwell, l'opinion publique se méfie de l'armée. De plus cette dernière demeure toujours responsable de ses activités envers le roi et non envers le Parlement. Celui-ci doit prendre ses précautions dont la plus étonnante est le «Mutiny Act». Voté annuellement, il légalise l'existence de l'armée pendant le terme d'une année, sans plus ${ }^{15}$. Le contrôle des finances de l'armée par le parlement et les pressions qu'il exerce pour obtenir des coupures à la moindre occasion rendent l'armée vulnérable aux assauts de ses détracteurs et limite sa marge de manoeuvre.

L'armée régulière britannique est numériquement modeste, en regard des normes européennes, notamment pendant les guerres napo-

14 John Sweetman, War and Administration. The Significance of the Crimean War for the British Army (Scottish Academic Press, 1984), 10.

15 E. E. Curtiss, The Organization of the British Army in the American Revolution (Oxford, Oxford University Press, 1926), 54. 
léoniennes qui voient le gonflement important des effectifs impliqués dans les combats. De plus, après la signature des traités de paix, invariablement, plusieurs régiments sont démembrés et presque toutes les nouvelles formations nées des besoins de la guerre sont licenciées. Les officiers moins expérimentés et réduits à l'inaction touchent alors la demi-solde.

Les observations précédentes entraînent un ensemble de caractéristiques avec lesquelles doit composer la gentilhommerie canadienne.

\section{2 - Des obstacles structuraux pour la gentilhommerie canadienne}

Il existe quatre moyens pour obtenir une promotion dans l'armée britannique: l'ancienneté, le recrutement («recruiting for rank»), le brevet et enfin l'achat. La première méthode peut se passer de commentaire. Le recrutement ne joue qu'en temps de guerre lorsque les rangs de l'armée doivent être augmentés. La troisième méthode, celle du brevet, consiste à promouvoir, par décision des autorités supérieures, un groupe d'officiers d'un échelon de la hiérarchie à l'échelon supérieur. En dépit de ses effets négatifs, dont les plus importants sont de retarder le développement d'une armée véritablement professionnelle ${ }^{16}$ et d'encourager un clivage social, la pratique de l'achat des commissions se perpétue jusqu'à la seconde moitié du dix-neuvième siècle, parce qu'elle épargne à l'État des sommes énormes ${ }^{17}$.

L'achat de commissions a une grande influence sur la constitution du corps des officiers de l'armée. Ce marché des grades joue contre la gentilhommerie canadienne qui doit rencontrer sur ce terrain l'aristocratie de l'empire. Il en coûte fort cher pour obtenir ainsi ses galons. Un officier ayant acheté toutes ses commissions, depuis le rang d'enseigne jusqu'à celui de lieutenant-colonel d'un régiment de ligne, doit débourser 3500 livres sterling pendant la période de notre enquête ${ }^{18}$, somme que plusieurs gentilshommes anglo-écossais n'hési-

16 Notons que l'artillerie, la marine et le corps des ingénieurs militaires ont pu éviter les charges vénales.

17 Ce sujet reste difficile à étudier en raison des pratiques auxquelles s'adonnaient les officiers en marge des règlements officiels. Voir en particulier Anthony Bruce, The Purchase System in the British Army (London, Royal Historical Society, 1980), 3-4.

18 De 1765 à 1811 , les prix officiels demeurent les mêmes, il faut donc avoir dépensé en tout: 400 livres pour une commission d'enseigne, 550 pour une de lieutenant, 1500 pour une de capitaine, 2600 pour une de major et 3500 pour une de lieutenant-colonel. T. Egerton, $A$ Treatise on Military Finance... (Londres, the Military Library near Whitehall, 1796), 48-51, et Horse Guards, General Regulations and Orders for the Army Adjudant Generals Office (Londres, 1811), 32. En 1854, les prix officiels n'ont guère varié: 450 livres pour une commission d'enseigne, 700 pour une de lieutenant, 1800 pour une de capitaine, 3200 pour une de major et 4500 pour une de lieutenant-colonel. Anthony Bruce, op. cit., 177-178. 
tent pas à dépasser en dépit du règlement ${ }^{19}$. De plus, le militaire qui ne veut pas verser davantage risque de se voir rejeter par ses collègues officiers $^{20}$. Le cas des Salaberry illustre bien les difficultés quasi insurmontables de ce système pour l'élite coloniale. Dans une lettre adressée à Louis de Salaberry au sujet de son fils Charles-Michel, le prince Edward, futur duc de Kent et de Strathearn, écrit:

I know it is the firm intention of the Duke of York [commandant en chef de l'armée] that no one shall be placed to his [CharlesMichel] injury in a company which should fall to him of right, in his Battalion, though one may succeed by purchase. You know that this infortunately cannot be avoided in our service by one who has not himself the means of purchase. ${ }^{21}$

Deux ans plus tard, c'est le secrétaire militaire en poste à Halifax qui vient rappeler cette même réalité à Charles-Michel:

His Royal Highness [le duc de Kent] had obtained a promise in your favor from the Duke of York, that no one should be promoted to your prejudice unless the vacancy should be occasioned by purchase, when, however mortifying it may appear, yet the custom and general rule of the service has established the practice beyond the possibility of its being obviated. ${ }^{22}$

Sous le régime français, ce système d'achat a cours dans l'armée de terre mais il est officiellement interdit dans les compagnies franches de la Marine. Jay Cassel, dans sa thèse de doctorat, relève seulement trois achats de commission chez les officiers de l'état-major des compagnies franches à l'époque de la Nouvelle-France ${ }^{23}$. La fréquence de ce mode de promotion distingue l'armée britannique des autres armées européennes aux XVIII ${ }^{e}$ et $\mathrm{XIX}^{\mathrm{e}}$ siècles. Elle accorde aux aristocrates bien nantis une mainmise sur l'armée qu'aucun autre groupe social n'est en mesure de lui disputer ${ }^{24}$. Ainsi, la carrière d'un Lord Brudenell (le futur Earl of Cardigan) qui obtient le grade de

19 Tous les officiers qui acceptent ou versent une somme supérieure à celles prescrites par le règlement dans une transaction visant à vendre ou à acheter une commission sont susceptibles de la perdre et d'être cassés en cour martiale. Horse Guards, op. cit., 36.

20 Edward M. Spiers, The Army and Society, 1815-1914 (Londres, Longman, 1980), 16.

21 Le prince Edward à Louis de Salaberry, Halifax, 30 avril 1798. William James Anderson ed., The Life of F.M., H.R.H. Edward, Duke of Kent (Ottawa et Toronto, Hunter, Rose and Company, 1870), 54.

22 James W. Gordon, secrétaire militaire à Charles-Michel de Salaberry, Halifax, 23 juin 1800, ibid., 66.

23 Jay Cassel, op. cit., 113.

24 Geoffrey Best, War and Society in Revolutionary Europe, 1770-1870 (Bungay, Suffolk, Fontana, 1982), 237. 
lieutenant-colonel en 1830, après seulement six ans de service ${ }^{25}$, n'offre aucun point de comparaison avec l'expérience des gentilshommes canadiens.

Le prix des commissions varie non seulement avec le grade convoité, mais également avec le type de régiment. Si le régiment est plus souvent sur les lignes de feu, ses grades sont offerts à moindre coût. Le $60^{\mathrm{e}}$ régiment accueille davantage de Canadiens, en bonne partie pour cette raison. Charles-Michel de Salaberry, Jean-Baptiste (1779-1833) et Michel-Louis (1785-1860) Duchesnay peuvent ainsi s'intégrer à l'armée régulière dans le $60^{\mathrm{e}}$ régiment. Nous savons que ce sont les parents de ce dernier qui lui ont fourni la somme nécessaire pour acheter une commission de lieutenant. Les explications données par Louis-René Chaussegros de Léry à son père au sujet de son impuissance à poursuivre une carrière militaire dans le $7^{\mathrm{e}}$ régiment résument nos propos. Il identifie trois obstacles:

...je ne crois pas être assez riche pour me soutenir dans le corps du prince... [de plus,] je n'ai pas la perspective d'avancement puisque je ne serai jamais en état d'acheter une compagnie et qu'à la paix le $2^{\mathrm{e}}$ bataillon sera sans doute réformé et avec lui les nouveaux officiers... et qu'enfin j'ai 33 ans [et] qu'il est un peu tard [pour] se mettre à la queue d'un régiment. ${ }^{26}$

Le corps des officiers forme un groupe favorisé par les autorités et son renouvellement s'effectue, pour une bonne part, dans les mêmes familles ${ }^{27}$. Le groupe est aussi jaloux de ses privilèges. Le gonflement des effectifs par l'incorporation de nouveaux officiers ou d'officiers de nationalité étrangère éveille toujours la susceptibilité des anciens. Pendant la guerre d'Indépendance américaine, par exemple, des hommes provenant de l'extérieur des cadres ordinaires de l'armée sont admis directement au rang d'officier dans des régiments non réguliers, à la condition qu'ils recrutent un nombre suffisant de soldats. Ce n'est que l'impérieuse nécessité de combler les rangs dégarnis de l'infanterie qui fait fléchir les réticences des officiers réguliers. Mais les responsables de cette politique sont conscients que la mesure irrite les vétérans, qui y voient un dangereux précédent, un accroc aux règles

25 Edward M. Spiers, op. cit., 16; voir également Gwyn Harris-Jenkins, The Army in Victorian Society (Toronto, University of Toronto Press, 1977), chapitre 3.

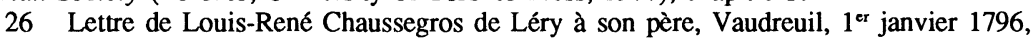
Pierre-Georges Roy, Inventaires des Papiers de Léry conservés aux Archives de la province de Québec (Québec, 1939-1940), 2: 187-188.

27 Michael Glover, Wellington's Army in the Peninsula, 1808-1814 (New York, Hippocreme Books, 1977) 36. 
établies $^{28}$. Le problème se pose avec les Loyalistes qui sont insatisfaits des emplois qui leur sont offerts dans l'armée, la rigidité du système ne permettant pas au cabinet anglais de North de répondre à leurs attentes ${ }^{29}$.

Seul le $60^{\mathrm{e}}$ régiment est ouvert aux officiers étrangers mais il est plus exposé au feu de l'ennemi que les autres unités de la GrandeBretagne. À plusieurs reprises, le «War Office» condamne des déserteurs à servir dans ce régiment plutôt que d'avoir recours à la flagellation $^{30}$, ce qui le rend encore moins attrayant pour les aspirantsofficiers.

Le système de patronage, présent dans plusieurs institutions de cette époque, est un élément fondamental dans l'armée, qui, dans ce cas, fonctionne à l'échelle de l'empire britannique ${ }^{31}$. Les familles canadiennes-françaises sont coupées des sources de patronage après 1760. Guy Carleton est le gouverneur qui fait le plus pour leur procurer des commissions dans l'armée. Toutefois, il obtient peu de chose, même lorsque, pour gagner l'appui de la classe seigneuriale durant la guerre de l'Indépendance américaine, les autorités se montrent plus généreuses. C'est le duc de Kent qui est le plus réceptif aux demandes de la gentilhommerie canadienne. Mais, compte tenu du petit nombre d'officiers canadiens dans l'armée et de la durée de leur présence, il est évident que le gros de la gentilhommerie est resté à l'écart des réseaux de patronage.

$\mathrm{Du}$ point de vue de son utilisation stratégique, l'armée régulière constitue le fer de lance de toutes les opérations d'envergure en temps de guerre sur le continent nord-américain ou ailleurs; en permanence, l'armée tient garnison aux quatre coins de l'empire. Il s'ensuit un système de rotation des effectifs à l'échelle américaine et impériale. Cette habitude de veiller aux déplacements des troupes renforce l'efficacité des soldats et des officiers tout en améliorant la discipline et, selon les autorités militaires, contribuerait à empêcher les nombreuses désertions en Amérique ${ }^{32}$.

28 E. E. Curtiss, op. cit., 71.

29 Paul H. Smith, Loyalists and Redcoats: a Study in British Revolutionary Policy (Williamsburg, University of North Carolina Press, 1964), 78.

30 Entre 1788 et 1792 , le régiment recevra ainsi pour des incartades plus de 300 hommes. Voir David Pimlott, The Administration of the British Army, 1783-1793, thèse de doctorat (histoire), Leicester, Université de Leicester, 1976, 228-230.

31 Gilles Paquet et Jean-Pierre Wallot ont bien montré l'importance du patronage pour le système politique du Bas-Canada. Ce n'est toutefois qu'une partie de l'ensemble. Patronage et pouvoir dans le Bas-Canada (1794-1812) (Montréal, Les Presses de l'Université du Québec, 1973).

32 La rotation des effectifs ne semble pas avoir connu beaucoup de popularité en Nouvelle-France. Pour la plupart, les officiers canadiens ne connaîtront que leur colonie comme 
Cette particularité du fonctionnement de l'armée britannique a pour les gentilshommes canadiens des conséquences fâcheuses puisqu'ils doivent suivre leur régiment au hasard de ses affectations ${ }^{33}$. Les membres des familles Fleury Deschambault, Juchereau Duchesnay, Salaberry, Saint-Ours et Vassal de Monviel sont amenés, certainement plus qu'ils ne le désirent, à servir dans le cadre impérial ${ }^{34}$.

En résumé, sous le régime britannique, la seule voie pour une véritable carrière militaire, avec tous les bénéfices et privilèges économiques (salaire, pension, concessions de terre, etc.) et sociaux (obtention de grade ou de décoration militaire et insertion dans un réseau d'alliances) qui s'y rattachent, est celle de l'armée régulière métropolitaine. Cependant, très peu de familles canadiennes associées jadis au métier des armes en tirent profit. Bref, l'élite militaire canadienne-française n'a pas de rôle à jouer dans l'armée régulière, dont la vocation est impériale.

\section{B - Milices et corps provinciaux au Canada}

Deux solutions de rechange s'offrent à la gentilhommerie canadienne sous le régime britannique: la milice et les corps provinciaux. S'il n'y a pas mobilisation, l'appartenance à la milice ne signifie souvent rien de plus que de voir son nom figurer sur une liste nominale. Quant aux corps provinciaux, leur nature varie selon l'époque et les circonstances. Trois problèmes de fond nuisent à la gentilhommerie dans son rôle militaire au Canada: l'absence d'une véritable organisation militaire coloniale qui soit permanente, le manque de plan d'ensemble de défense impériale pour l'Amérique et l'incapacité de la gentilhommerie à jouer un rôle mobilisateur au sein de la société coloniale.

théâtre d'activité; il leur est même arrivé de refuser une rotation Canada-Plaisance-Acadie. Christopher J. Russ, Les troupes de la Marine, 1683-1713, mémoire de maitrise (histoire), Université McGill, 1971, 156-157). En 1698, lorsque la force des compagnies franches sera réduite, ils tiendront à rester dans la colonie. Évidemment, cela n'exclut pas le devoir d'effectuer des missions de plus ou moins longue durée dans d'autres milieux coloniaux ou, pour les jeunes officiers, de chercher des emplois à l'extérieur. Au sujet des mouvements des forces britanniques en Amérique, voir John Shy, Toward Lexington (New Jersey, Princeton University Press, 1965).

33 Jusqu'aux années 1870 , la Grande-Bretagne impose à ses officiers de servir environ les deux-tiers de leur carrière à l'étranger. Voir Theodore Ropp, War in the Modern World (Durham, N. C., Duke University Press, 1959), 127-128.

34 Saint-Ours déploie beaucoup d'ingéniosité pour rester au Canada sans renoncer à sa carrière. Les trois frères Salaberry ont pour objectif avoué de faire carrière au Canada même s'ils doivent poursuivre la quête de grades dans l'ensemble de l'empire. 


\section{1 - 1775-1783: la guerre de la révolution américaine}

Comme nous le savons, la métropole met un certain temps à évaluer l'ampleur de la révolte des Treize Colonies. Dépassés par les événements, Lord Dartmouth, secrétaire aux colonies, et son entourage n'ont pas de stratégie adaptée à ce contexte particulier sinon de s'en remettre à une levée en masse improvisée des Canadiens. Mise à part l'acceptation par le général Gage de l'offre d'Allan McLean de lever le «Royal Highlands Emigrants» en juin 1775, les corps de Loyalistes, qui prennent forme peu à peu, ne reçoivent une politique d'appui véritable que plus tard dans le conflit ${ }^{35}$. Au mois de juillet 1775, Dartmouth demande au gouverneur Carleton de lever une petite armée de 6000 Canadiens pouvant servir d'infanterie légère, soit en assistant les troupes régulières, soit en agissant seule, selon les circonstances $^{36}$. Pour la première fois depuis la Conquête, la «noblesse canadienne» peut escompter servir les autorités britanniques en assumant le commandement des nouvelles troupes canadiennes. Du moins, les dispositions avantageuses sur les plans sociaux et politiques de l'Acte de Québec lui permettent-elles d'entretenir des espérances. Carleton souhaitait depuis longtemps la participation de la gentilhommerie à l'armée, avec des avantages identiques à ceux des autres sujets britanniques. Il plaide leur cause en ces termes auprès de ses supérieurs:

The Canadians have testified to me the strongest Marks of Joy and Gratitude and Fidelity to The Arrangements made at home in their Favor [l'Acte de 1774], a Canadian Regiment would complete their Happiness... and I am convinced their Fidelity and Zeal might be depended on; should this Measure be at lenght adopted (which I have long Recommended)... their appointments should be the same as the rest of the Infantry, with half pay in Case they should be reduced. ${ }^{37}$

Dans ses instructions au gouverneur, Dartmouth refuse les avantages réclamés par Carleton pour les Canadiens: les commissions d'officier ne permettent pas à leur titulaire de réclamer la demi-solde, ne sont valides qu'en Amérique et sont inférieures à celle que détient le plus jeune officier de l'armée britannique d'un grade équivalent ${ }^{38}$. Le

35 Voir notamment à ce sujet l'ouvrage de Paul H. Smith, op. cit.

36 Darmouth à Carleton, Whitehall, $1^{\text {er }}$ juillet 1775, Public Record Office, C.O. 42, vol. 34 , f.112.

37 «Extract of General Carleton's Answer to General Gage», Québec, 20 septembre 1774, Public Record Office, C.O. 42, vol.33.

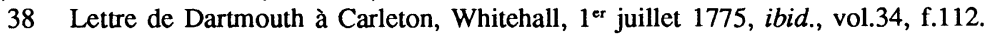


Secrétaire insiste, le 24 juillet, en répétant les conditions d'enrôlement des officiers canadiens ${ }^{39}$.

De plus, aux yeux de Londres, la valeur de la gentilhommerie tient à sa capacité de susciter l'adhésion du reste de la société canadienne. Or il existe une irréductible opposition entre la gentilhommerie et le peuple en ce qui a trait à la défense de la colonie. Ce dernier abhorre l'armée et préfère la milice. Témoin ce capitaine britannique qui, prenant en compte ces réticences, estime qu'il est sage de maintenir l'appellation de milice pour la population, peu importe la réalité que recouvre vraiment ce terme ${ }^{40}$. À l'opposé, le gouverneur Carleton constate que, pour une partie de l'élite canadienne, accomplir son devoir militaire dans la milice ne manque pas d'être perçu comme déshonorant. C'est ce qui s'est d'ailleurs produit à l'occasion de la levée du corps expéditionnaire canadien contre Pontiac ${ }^{41}$.

C'est dans ce contexte que Joseph-Dominique-Emmanuel Lemoyne de Longueuil (1738-1807) et François-Marie Picoté de Belestre prennent le pari de la reconnaissance politique par les nouvelles autorités en acceptant les commandements au fort SaintJean en 1775. Nous connaissons l'insuccès de la mobilisation des Canadiens à cette occasion. Peut-être est-ce à cause de la situation stratégique précaire des forces britanniques? Ou encore à cause de liens sociaux ou économiques trop lâches entre la gentilhommerie et la paysannerie ${ }^{42}$ ? Chose certaine, Longueuil et Belestre se présentent au peuple dépouillés de tout moyen de coercition pour l'embrigader.

La première ordonnance «qui règle les Milices de la Province de Québec» date de 1777. À son sujet, précisons que nous ne nous intéressons pas au rôle nominal de la milice, essentiellement une force civile d'appoint dans lequel se retrouvent tous les groupes sociaux. $\mathrm{Ce}$ qui nous importe, c'est la milice active dont traite l'article VI de l'ordonnance:

En temps de guerre, de rébellion ou en autres cas urgents, suivant que les circonstances l'exigeront, des officiers, des sergents et

39 Lettre de Darmouth à Carleton, Whitehall, 24 juillet 1775. Cité par Ernest Cruikshank, A History of the Organization, Development and Services of the Military and Naval Forces of Canada from the Peace of Paris in 1763, to the Present Time (Ottawa, The Historical Section of the General Staff, 1919), 2: 63. En ce qui a trait à Dartmouth, pour comprendre ses réserves, il suffit de nous reporter à la première partie de cet article.

40 «Memorandum on Canadian Militia», capitaine Foy, 6 février 1778, ibid., 241.

41 Lettre de Carleton à Dartmouth, Québec, 20 novembre 1775, ibid., 134.

42 Rappelons aussi que sous le régime français, l'officier des compagnies franches de la Marine commande indirectement le milicien canadien. 
miliciens, ainsi que l'ordonnera le Gouverneur, le Lieutenantgouverneur ou le commandant en Chef, seront tirés des différentes paroisses à l'endroit qui leur sera commandé, et serviront, quoique toujours comme Milice, conjointement avec les troupes de sa Majesté, sous la conduite et inspection des officiers qu'il plaira au Commandant en Chef de nommer à cet effet... ${ }^{43}$

Cette fois, cet article permet la participation de plus de 1000 Canadiens au plan de redressement de l'autorité britannique en Amérique du Nord. Toutefois, le gouverneur et général Carleton d'abord, puis le général Burgoyne établissent seulement trois compagnies de combat qu'ils placent sous la responsabilité de la gentilhommerie. Celle-ci répond à l'appel avec empressement. Le reste des miliciens actifs appelés pour le service s'occupent de soutenir l'armée en se chargeant particulièrement du transport des troupes et des provisions. Ils relèvent alors plutôt de gens d'affaires, tels DufyDesaunier ou les frères Lecompte Dupré, que de l'ancienne élite militaire.

Les trois maigres compagnies disparaissent à la fin des hostilités parce qu'elles n'existent que pour répondre aux besoins du moment. Comble de malchance, les officiers de deux de ces compagnies sont capturés en 1777 et incapables de toucher leurs émoluments parce qu'ils recouvrent la liberté moyennant l'interdiction de reprendre le service militaire.

L'arrivée du gouverneur Frederick Haldimand en remplacement de Carleton n'aide pas les choses. Après les insuccès de Burgoyne, aucun Canadien ne demeure sur le pied de guerre. Dans un premier temps, le nouveau gouverneur voit les choses du même oeil que Guy Carleton. Il favorise donc la constitution d'une formation de Canadiens forte d'un millier d'hommes commandée uniquement par des Canadiens dans un but tant social que stratégique. D'abord aider à restaurer l'influence de la noblesse sur le peuple et les Amérindiens pour permettre une juste distinction entre les classes. Ceci, dans l'esprit du gouverneur, ne manquerait pas de susciter l'obéissance et la subordination chez les classes sociales inférieures ${ }^{44}$. Cependant, Haldimand ne tarde pas à effectuer une volte-face, troublé par le danger que laisse planer sur le comportement des Canadiens l'entrée en guerre de la France aux côtés des États-Unis. Aussi, lorsqu'à

43 Arthur G. Doughty, Rapport concernant les travaux des archives publiques pour les années 1914 et 1915 (Ottawa, 1917), 72.

44 «Sketch of the Military State of the Province of Quebec», lettre de Haldimand à Germain, Québec, 25 juillet 1778, Archives nationales du Canada, MG 21, B 42, f.13-14. 
Londres le secrétaire d'État pour les colonies, Lord Germain, suggère à Haldimand la levée d'une force coloniale, ce dernier s'y oppose de peur de donner des armes à des sujets dont il appréhende le soulèvement ${ }^{45}$. Cette perception du climat politique lui commande de se méfier de toutes les classes canadiennes et de s'en remettre aux troupes régulières pour défendre la colonie.

Dans sa réponse à Lord Germain, le gouverneur souligne toutefois qu'il y aurait lieu de redonner à la gentilhommerie le rôle du commandement des guerriers amérindiens. Ainsi, les Lacorne et les Lorimier seront employés à assurer la collaboration des Amérindiens en raison des liens qu'ils entretiennent avec eux depuis le régime français. Leur réussite représente alors une certaine importance stratégique pour les autorités britanniques. L'alliance avec les autochtones, fort précieuse, permet d'effrayer la population civile de la frontière américaine en plus de disposer de renforts durant les combats.

Luc de Lacorne participe à la guerre de la révolution américaine à la tête d'un groupe d'Amérindiens à la bataille de Saratoga. Quant à Claude-Nicolas-Guillaume de Lorimier (1744-1825), il doit d'abord convaincre des autochtones de le suivre, puis, sans grade de l'armée régulière, obéir aux instructions du général Burgoyne. D'ailleurs plus sûrs de la reconnaissance de leur valeur stratégique ${ }^{46}$ que d'autres anciens officiers des compagnies franches de la Marine, Lacorne et Lorimier ont peut-être hésité sur le parti à prendre. Le premier échange sur un ton bienveillant avec le général Montgomery tandis que, lors de la prise de Montréal par les Américains en 1775, le second reçoit par l'entremise d'un agent amérindien quelques offres pour joindre les rangs de l'armée rebelle en qualité de major. C'est avec une louche insistance que Claude-Nicolas-Guillaume de Lorimier dénonce cet agent par la suite.

Si l'on ajoute les postes d'aide de camp provincial (Luc de Lacorne et Charles-Louis-Roch de Saint-Ours) et d'inspecteur des milices (Louis-Joseph Fleury Deschambault et Joseph-DominiqueEmmanuel Lemoyne de Longueuil), voilà le bilan de ce que récolte un groupe social, au premier chef intéressé par la défense de la colonie durant toute la durée de la guerre d'Indépendance. Aucune orga-

45 Lettre de Haldimand à Germain, 25 octobre 1780, Public Record Office, C.O. 42, vol.40, f.101-109.

46 Dans son journal, Claude-Nicolas-Guillaume prétend qu'il a dû reprendre du service immédiatement après avoir reçu une blessure, prié par le commandant du «Royal Highland Emigrants» de convaincre des Amérindiens d'appuyer le général Burgoyne en difficulté aux États-Unis. «Journal de de Lorimier», Abbé Verreau, Invasion du Canada, collection de mémoires recueillis et annotés (Montréal, Eusèbe Sénécal, 1873), 293. 
nisation militaire coloniale ne subsiste. Celle qui a connu le plus de succès, le régiment d'Allan Maclean, obtient une reconnaissance et est accepté dans la «ligne», c'est-à-dire comme un régiment de l'armée à part entière, pour devenir le $84^{e}$ régiment ou le «Royal Highland Emigrants». Cependant, il ne fait pas long feu. En raison de sa jeunesse, les réductions d'effectifs qui touchent plusieurs régiments de l'armée à la fin du conflit ne l'épargnent pas. Remarquons toutefois que la gentilhommerie canadienne n'a pas su tirer parti non plus de cette unité. Seul Charles-Louis-Roch de Saint-Ours y a obtenu une commission.

\section{2 - 1783-1812: les expériences}

Si quelques membres de la gentilhommerie ont l'occasion de s'enrôler dans l'armée régulière, reçoivent divers témoignages politiques de reconnaissance, décrochent des postes permanents au département des affaires indiennes (interprète, agent résidant et surintendant), la plupart des officiers des trois compagnies canadiennes doivent attendre 1787 pour récolter une demi-solde et des concessions de terre. Cette reconnaissance tardive des services militaires rendus ne survient qu'en 1787, au moment où lord Dorchester se soucie de rendre plus efficace la milice dans des circonstances de tensions internationales. Il s'agit alors de trouver les mécanismes et de réaliser les compromis nécessaires pour la rendre active au moment désiré. Conscient de l'importance de l'aide que pourrait apporter la milice canadienne aux forces régulières britanniques ${ }^{47}$, le gouverneur souhaite que ces récompenses puissent convaincre les Canadiens qu'ils sont traités à égalité avec les autres sujets de la Couronne. Toutefois, les succès mitigés des premières tentatives de rassemblement, en 1794, 1801 et 1807 , font douter d'un support efficace de la milice à l'armée.

Un projet plus important et plus prometteur pour une carrière militaire qu'une milice, même mobilisée, est annoncé par les autorités supérieures de l'armée en 1794. Il s'agit de la mise sur pied d'un régiment provincial, le «Royal Canadian Volunteers». Sa mission principale est de veiller sur le Bas et le Haut-Canada parce que la Grande-Bretagne, en guerre avec la France, a rappelé d'Amérique bon nombre de soldats. La raison de ce retournement dans la politique de défense s'explique par le doute qu'entretient le secrétaire d'État Henry Dundas sur une levée efficace de la milice canadienne et par la nécessité d'augmenter les forces de la colonie ${ }^{48}$.

47 Dorchester à Sydney, Québec, 8 novembre 1787, Public Record Office, C.O. 42, vol.51, f.204; du même au même, Québec, 28 février 1787, ibid., vol.50, f.68.

48 Lettre de Dundas à Dorchester, Whitehall, 15 février 1794, ibid., vol.98, f.81. 
Le premier bataillon s'adresse avant tout à la gentilhommerie canadienne, l'autre à l'élite militaire anglo-écossaise du Haut-Canada. Le régiment est, d'abord et avant tout, un essai qui dure jusqu'en 1802. Il nous a été possible de reconstituer l'expérience significative d'un membre important de la gentilhommerie dans cette aventure.

Joseph-Dominique-Emmanuel de Longueuil prend la tête du premier bataillon en qualité de lieutenant-colonel. Toutefois, il semble bientôt éprouver quelques ennuis à s'intégrer à cette nouvelle structure militaire. Ainsi, s'il a la chance de se soustraire au paiement du «halfmounting $\gg^{49}$, charge communément assumée par les officiers commandants des régiments réguliers, il doit toutefois se justifier devant les reproches de ses supérieurs concernant les «extraordinaires $»^{50} \mathrm{de}$ son bataillon qui excèdent les normes établies. Plus tard, il demande la compréhension des autorités pour faire reconnaître sa situation particulière et celle de son régiment par rapport aux régiments réguliers quant à l'approvisionnement en vêtements de ses hommes. Il tente d'obtenir, à cet effet, un remboursement de 1000 livres anglaises. D'autre part, contrairement à l'usage de faire construire des baraquements, Longueuil se contente de louer un appartement pour y installer les tailleurs qui confectionnent les uniformes. À sa décharge, il affirme avoir déjà fourni «...le nécessaire [aux] soldats: en habits, culottes et bonnets» à ses propres frais. De plus, le lieutenant-colonel $\mathrm{du}$ «Royal Canadian Volunteers» doit s'en remettre à un sergent du $60^{\circ}$ régiment de l'armée régulière pour l'aider dans les parties comptables de ses fonctions. Enfin, la manière dont il distribue le bois de chauffage à ses soldats lui vaut de passer devant une cour d'enquête («Court of Inquiry»). Chez le reste de l'armée, le procédé permet habituellement un léger bénéfice pour les officiers. Toutefois, Longueuil s'y est mal pris. Appétit de gain trop grand ou tout simplement ignorance des procédures informelles? «Si j'avais jamais eu des ordres ou même un guide concernant mes devoirs envers mon régiment je n'y aurais jamais dévié», argumente-t-il dans une lettre au secrétaire militaire ${ }^{51}$. À l'occasion de son testament, il avoue que le décès récent du paie-maître du régiment le laisse sans moyen de récupérer l'argent que l'armée lui doit toujours, lui qui déclarait à l'été 1797 à son

49 Qui consiste à fournir à chaque soldat une paire de souliers, une chemise, un collet et quelques autres menus articles d'habillement.

50 Ce sont des dépenses qui ont trait à l'activité du bataillon, tels le transport et le chauffage.

51 Longueuil à Green, le 2 novembre 1801, Archives nationales du Canada, RG 8, I, A1, vol. 793, f.284-285. 
supérieur: «...je vous avoue que je suis las de payer, car tous tombent sur le régiment. $»^{52}$

L'existence du «Royal Canadian Volunteers» est menacé par le commandant en chef de l'Amérique du Nord britannique, le duc de Kent, qui veut embrigader la totalité des hommes des corps provinciaux tels ceux du bataillon de Longueuil «...on the new System as Fencibles $»^{53}$. Il obtient même, à son grand contentement, l'assentiment des soldats de la huitième compagnie du bataillon de Longueuil pour procéder aux changements. Mais dans ce projet de réaménagement de la participation canadienne à la défense de leur territoire, les officiers proviennent des forces régulières et l'activité du régiment s'étend désormais à toute l'Amérique du Nord britannique, et non plus seulement au Canada. Dans ces conditions, les gentilshommes canadiens sont pratiquement tous écartés. À la paix d'Amiens, des réductions aux forces de terre sont commandées dans l'empire. Le «Royal Canadian Volunteers» est alors démantelé. Or c'est la formule du duc de Kent qui est retenue lorsqu'il est question de mettre sur pied des unités coloniales avec le renouvellement des hostilités en Europe en 1803.

Le «Canadian Fencible Regiment of Infantry» naît en 1803, parallèlement à d'autres régiments de type identique à Terre-Neuve, en Nouvelle-Écosse et au Nouveau-Brunswick. Laissée pour compte, la gentilhommerie réclame pourtant son incorporation à un autre régiment $^{54}$ depuis la disparition du «Royal Canadian Volunteers». L'évolution de la composition du groupe des officiers des «Fencibles» (par les promotions, les affectations et les échanges, etc.) montre qu'il se rattache à l'ensemble des gradés de l'armée britannique quoique le régiment possède un statut moindre que les réguliers. Si ce genre d'organisation permet plus de souplesse stratégique, parce que plus mobile et constitué d'un personnel de commandement plus qualifié, il rencontre, malgré tout, un sérieux problème de recrutement. Le «Canadian Fencible» n'est d'ailleurs inscrit sur la liste des régiments actifs qu'en novembre 1808, non sans que l'on ait préalablement réduit à 400 recrues les exigences à son inscription.

52 Lettre de Longueuil au secrétaire militaire Green, 10 août 1797, Archives nationales du Canada, RG 8, I, A1, vol.793, f.272.

53 Lettre du prince Edward au lieutenant-général Hunter, Halifax, 28 mars 1800, ibid., vol.223, f.202.

54 Clément-Christophe-Anne Sabrevois de Bleury (1755-1827) se fera, par exemple, fort insistant auprès d'un officier britannique qu'il croit être en mesure de l'aider. Lettre de Sabrevois de Bleury au major Green, Boucherville, 27 septembre 1803, Archives nationales du Canada, RG 8, I, A1, vol.16, f.88; du même au même, Boucherville, 23 novembre 1803, ibid., f.117. 
D'autres projets demeurent lettre morte durant la période qui s'étend de la fin du «Royal Canadian Volunteers» jusqu'aux préparatifs de la guerre de 1812. L'un de ceux-là, qu'aide à réaliser le duc de Kent, vise à embrigader les Canadiens sous l'autorité du major John Robertson du $60^{\mathrm{e}}$ régiment $^{55}$. Avec les régiments de «Fencibles», sans la gentilhommerie et avec relativement peu de Canadiens, l'incorporation à l'armée fait un pas en avant. L'expérience du «New Brunswick Fencible Infantry» le prouve, lui qui est incorporé à la ligne (104 ${ }^{\mathrm{e}}$ régiment).

Il faut envisager une autre solution que les régiments de «Fencibles» si l'on désire la participation des Canadiens français à leur défense. Le gouverneur Prévost a l'ordre de lever un régiment, pour 1812 , dans lequel il s'efforcera de ménager une place à la gentilhommerie. Pour en assurer un commandement efficace, il recherche un officier ayant une origine canadienne qui se distingue par son zèle, son adresse et son intelligence ${ }^{56}$. En plus de réunir toutes ces qualités, Charles-Michel de Salaberry possède en plus l'expérience du service britannique comme la plupart des officiers des Voltigeurs.

\section{3 - La guerre de 1812}

Avec ménagement et succès, l'administration coloniale appelle sous les drapeaux la masse des Canadiens français qui forment les Voltigeurs et la milice d'élite et incorporée. L'organisation militaire pour la guerre de 1812 est d'une grande complexité, expression de la diversité des intérêts en présence dans la colonie ${ }^{57}$. Le cas des Voltigeurs en fournit un bon exemple. Les officiers de cette unité provinciale sont payés par l'armée britannique mais servent sous la loi coloniale de la milice du Bas-Canada ${ }^{58}$. De plus, à l'exception de

55 Stephen George Roberts, Imperial Policy, Provincial Administration and Defence in Upper Canada, 1796-1812, thèse de doctorat (histoire), Université d'Oxford, 1975, 284.

56 E. Cruikshank, «Record of the Services of Canadian Regiments in the War of 1812: Part II - The Glengarry Light Infantry», Selected Papers from the Transactions of the Canadian Military Institute, 6 (1894-1895): 9.

57 L'antagonisme des groupes sociaux, du monde rural et urbain, des métropolitains et des coloniaux, des ethnies, du civil et du militaire, se réflètent dans les organisations militaires et para-militaires. Même l'adjudant général des milices n'arrive pas à énumérer les différentes forces sous ses ordres dans ce message qu'il adresse à ses subordonnés: «... tous les officiers commandant les différents bataillons de milice, ainsi que ceux des corps de volontaires, d'artillerie, de cavalerie, Light Infantry, ou corps légers, compagnie de chasseurs ou tout autre corps de milice incorporée quelconque...». "General Orders», Québec, 19 juin 1813, Archives nationales du Canada, RG 9, 1A3, vol. 2.

58 William Wood, «Conditions for raising a Corps of Light Infantry», Québec, 15 avril 1815, Select British Documents of the Canadian War of 1812 (Toronto, The Champlain Society, 1928), 3,2: 653 . 
Salaberry, les officiers: «... will rank junior to all Officers of their respective Ranks in the Line or Fencibles: and are not to be considered as having a Claim to Half Pay or any other Allowance in right of their Commissions, whenever it may be disbanded.» ${ }^{59}$ Une constante de l'organisation militaire britannique, ces dispositions protègent les officiers déjà en place et ménagent le trésor de guerre.

Riche de ses expériences passées, Londres ne compte plus seulement sur la gentilhommerie pour enrégimenter la population comme le montrent les nominations du général Prévost pour les différents postes d'officier. La gentilhommerie s'impose malgré tout au gouverneur aussitôt après son arrivée au Canada par ses réseaux d'alliance et par sa présence auprès du pouvoir politique. Désireux de connaître les hommes pouvant lui être utiles pour la défense de la colonie, Prévost s'adresse à l'adjudant général des milices qui lui communique alors une liste de douze noms dont huit ont fait partie du «Royal Canadian Volunteers» et neuf peuvent être rattachés à la gentilhommerie. Ainsi, cette dernière réussit à s'assurer d'une présence importante parmi les officiers des milices d'élite et incorporées et des autres unités coloniales. Elle contribue également au lien, si ténu soit-il, qui se tisse entre l'armée métropolitaine et la société coloniale pendant la guerre contre les Américains. L'exemple de Vassal de Monviel le montre bien. En octobre 1811, il remplace un François Baby vieillissant au rang le plus élevé de la milice, celui d'adjudant général des milices du Bas-Canada. Monviel allie l'expérience de l'armée régulière à celle des organisations coloniales. Quant à Joseph Fleury Deschambault, qui sert lui aussi quelques années dans les régiments de ligne, il réussit à faire incorporer un groupe de miliciens sédentaires pour former le $7^{\mathrm{e}}$ bataillon de milice d'élite.

Les paragraphes précédents ne doivent pas faire illusion sur la façon dont les forces militaires potentielles de la colonie sont gérées par les dirigeants métropolitains et coloniaux. L'incapacité de mobilisation demeure puisque l'exemple de Louis-Joseph Fleury Deschambault constitue davantage une exception que la règle. Son unité a une existence de très courte durée. Quant aux Voltigeurs, qui connaissent le succès dans les opérations militaires et dans le recrutement, ils ne survivent pas à la fin de la guerre en dépit des espoirs de ses jeunes officiers ${ }^{60}$.

59 Ibid.

60 À propos des Voltigeurs et du recrutement durant la guerre de 1812, voir Michelle Guitard, Histoire sociale des miliciens de la bataille de la Châteauguay (Ottawa, Direction des lieux et des parcs historiques, Parcs Canada, 1983) et Luc Lépine, La participation des Canadiens français a la guerre de 1812, mémoire de maîtrise (histoire), Université de Montréal, 1986. 
Dans l'ensemble, la guerre de 1812 ne modifie pas la politique métropolitaine concernant la gentilhommerie canadienne et les forces armées. Malgré quelques succès dans les combats, aucune structure permanente d'enrôlement des Canadiens n'émerge de ce conflit. De plus, la perte du soutien inconditionnel du gouverneur pour les affaires militaires ajoute à l'étiolement de la force sociale de la gentilhommerie. L'exclusivité du recours à la force reste entre les mains des troupes régulières britanniques installées en garnison dans la colonie. Toujours convaincus que les États-Unis constituent une menace pour l'avenir, les dirigeants britanniques orientent plutôt leur choix stratégique vers des entreprises de fortification, qui nécessitent moins de troupes, dans un plan d'ensemble résolument axé sur la défensive.

\section{CONCLUSION}

Pour mettre à contribution la population canadienne pour la défense de la colonie et prêter main-forte à l'armée régulière, les autorités politiques de la métropole et les administrateurs coloniaux recourent, comme en Europe, à la gentilhommerie. Plusieurs familles de l'ancienne «caste militaire» du régime français ont ainsi l'occasion de renouer avec le métier des armes. Or ce rôle militaire, la gentilhommerie canadienne doit l'assumer à l'extérieur de l'armée britannique.

Les quelques tentatives de carrière dans l'armée régulière nous róvèlent les difficultés de l'entreprise: le patronage insuffisant, le manque de ressources financières, les risques de servir dans des régiments plus exposés, l'absence d'expérience, etc. Les conditions d'emploi dans l'armée régulière britannique, telles qu'elles se présentent, semblent ne pouvoir servir que modestement au dynamisme de la gentilhommerie dans la société coloniale. Certes, il y a des occasions où le lien avec l'armée impériale procure des dividendes (demi-solde, poste important dans la milice, etc.), mais ils sont insuffisants tant en nombre qu'en qualité. Au Canada, l'absence de structure militaire permanente de concert avec les réticences de la population à suivre un «ordre guerrier» incapable de l'enrégimenter, forment une partie du décor (certes quelque peu modifié avec les ans mais qui reste solidement en place jusqu'en 1815) dans lequel s'empêtrent les carrières militaires de la gentilhommerie canadienne sous le régime britannique.

À ce chapitre, la gentilhommerie échoue et ne prouve donc pas son importance stratégique. Mais les politiques inconsistantes ainsi que l'incohérence entre les objectifs et les moyens utilisés par les dirigeants britanniques désavantagent ce groupe social. Ils se montrent incapables de mettre sur pied une véritable organisation pour accueillir 
les officiers et les aspirants officiers canadiens. C'est que la volonté de la Grande-Bretagne de préserver son monopole de l'emploi de la force dans ses colonies ne permet pas certaines concessions.

La gentilhommerie canadienne joue ainsi un rôle imprécis et de second ordre dans la défense de l'imperium britannique. Parce qu'elle ne possède pas le poids social et économique nécessaire, elle n'est pas en mesure, lorsque l'occasion lui en est donnée, de remplir les devoirs qui incombent normalement à la noblesse britannique et de réaliser ses aspirations. En dernière analyse, l'étude des organisations de défense nous suggère que la déstructuration de l'univers institutionnel militaire prépare l'accélération du déclin de la gentilhommerie canadienne. En effet, cette déstructuration rend impossible sa dynamisation en l'exposant à la prépondérance de la gentilhommerie de l'empire britannique sur le même terrain d'action.

Les aléas de la gentilhommerie canadienne sont le fait non seulement d'une politique militaire nouvelle mais aussi d'une structure d'organisation différente.

Les impératifs de défense militaire des intérêts métropolitains en Amérique du Nord ne se bornent pas à assigner un rôle, par ailleurs effacé, à la gentilhommerie. Ils déterminent, ou contribuent à déterminer d'autres éléments de la socio-économie canadienne bien au-delà de la trame politique où l'on s'est contenté jusqu'ici de l'observer. À notre avis, l'étude de l'activité militaire au Canada recèle un potentiel heuristique énorme si elle se donne la peine de dépasser l'histoire bataille ${ }^{61}$.

61 À titre d'exemple, mentionnons André Charbonneau, Yvon Desloges et Marc Lafrance, Québec, ville fortifiée du XVII' au XIX' siecle (Ottawa, Éditions du Pélican et Parcs Canada et le Centre d'édition du gouvernement du Canada, 1982); Fernand Ouellet, «The British Army of Occupation in the St.Lawrence Valley, 1760-1774: the Conflict Between Civil and Military Society", R. A. Prete et A. H. Ion, Armies of Occupation (Waterloo, Ont., 1984); enfin Gilles Paquet et Jean-Pierre Wallot traitent assez longuement des dépenses militaires pour le Bas-Canada et en reconnaissent l'importance dans Patronage et pouvoir dans le Bas-Canada (1794-1812), op. cit. 